ISSN 0103-5150

Fisioter. Mov., Curitiba, v. 25, n. 2, p. 263-272, abr./jun. 2012 Licenciado sob uma Licença Creative Commons

\title{
Lombalgia crônica: comparação entre duas intervenções na força inspiratória e capacidade funcional
}

\author{
Chronic low back pain: comparison of two interventions \\ in inspiratory strength and functional capacity
}

\section{Flávia Tomé ${ }^{[a]}$, Caroline Borges Ferreira ${ }^{[b]}$, Rodrigo Junior Becker Cornelli ${ }^{[c]}$, Alberito Rodrigo de Carvalho ${ }^{[\mathrm{d}]}$}

[a] Fisioterapeuta Graduada pela Universidade Estadual do Oeste do Paraná (UNIOESTE), Cascavel, PR - Brasil, e-mail: flaviatomefisio@yahoo.com.br

[b] Fisioterapeuta Graduada pela Universidade Estadual do Oeste do Paraná (UNIOESTE), Cascavel, PR - Brasil, e-mail: carol-bferreira@hotmail.com

[c] Fisioterapeuta Graduado pela Universidade Estadual do Oeste do Paraná (UNIOESTE), Cascavel, PR - Brasil, e-mail: rodrigojrnelli@hotmail.com

[d] Fisioterapeuta, Mestrando no programa de Ciências do Movimento Humano (UFRGS), docente do curso de Fisioterapia da Universidade Estadual do Oeste do Paraná (UNIOESTE), Cascavel, PR - Brasil, e-mail: alberitorodrigo@gmail.com

\section{Resumo}

Introdução: A dor lombar crônica apresenta alta incidência, grandes custos sociais e pessoais e esforços terapêuticos muitas vezes frustrantes. Faz-se necessário, então, avaliar os recursos fisioterapêuticos para entendê-los melhor e fundamentá-los cientificamente. Objetivos: Comparar os efeitos entre duas intervenções fisioterapêuticas sobre a força muscular respiratória (FMR) e a capacidade funcional (CF) em pacientes com lombalgia crônica. Materiais e métodos: Série de casos cuja amostra ( $n=10)$, composta por portadores de dor lombar crônica, foi dividida aleatoriamente em grupo controle, que recebeu fisioterapia convencional (eletroanalgesia, massoterapia e flexibilização), e grupo experimental, que recebeu a cinesioterapia combinada (composta por técnicas de isostretching + treinamento sensório-motor aquático). A mensuração da CF, realizada pelo teste de caminhada de seis minutos (TC6), e da FMR, pelas pressões máximas inspiratória (Pimáx) e expiratórias (Pemáx) por meio de manuvacuometria, aconteceu antes ( $\Delta \mathrm{INI}$ ) e após $(\triangle$ FIN) as intervenções, que duraram sete semanas, com três sessões semanais. As comparações foram feitas pelo Mann-Whitney e Wilcoxon $(\alpha=0,05)$. Resultados: No GE, observou-se melhora, intragrupo, na Pimáx 
( $\mathrm{p}=0,0164)$, Pemáx $(\mathrm{p}=0,0227)$ e TC6 ( $\mathrm{p}=0,0092)$, e, no GC, apenas no TC6 ( $\mathrm{p}=0,018)$. Nas comparações intergrupo, obteve-se Pimáx e Pemáx semelhantes na $\Delta I N I$, mas diferentes na $\Delta$ FIN ( $p=0,0166 ; p=0,0045)$; e no TC6, observou-se diferença significativa apenas na $\Delta \mathrm{INI}(\mathrm{p}=0,0484)$. Conclusão: 0 isostretching e treinamento sensório-motor aquático foram eficazes na melhora da FMR e da CF, e o tratamento fisioterapêutico convencional foi efetivo apenas na melhora da CF.

Palavras-chave: Dor lombar. Músculos respiratórios. Terapia por exercício. Propriocepção.

\section{Abstract}

Introduction: Chronic low back pain has a high incidence, large social and personal costs and therapeutic efforts are often frustrating. Is necessary evaluated the physical therapy resources to understand better and substantiating scientifically then. Objectives: To compare the effects of two physical therapy interventions on respiratory muscle strength (RMS) and functional capacity (FC) in patients with chronic low back pain. Materials and methods: The sample $(n=10)$ was comprised of patients with chronic low back pain randomly divided into the control group (CG), which received conventional physical therapy (electroanalgesia, massage and stretching), and the experimental group (EG), which received the combined kinesiotherapy (composed of isostretching and aquatic sensoriomotor training). The measurement of the CF test performed by the six minute walk test (6MWT) and the RMS for inspiratory maximal pressure (IMP) and expiratory (MEP) by manovacuometry, which happened before ( $\triangle I N I)$ and after ( $\triangle F I N)$ interventions, with the duration of seven weeks, three times a week. Comparisons were made by Mann-Whitney and Wilcoxon tests $(\alpha=0.05)$. Results: The EG showed improvements intra-group in IMP ( $p=0.0164), E M P(p=0.0227)$ and 6MWT $(p=0.0092)$, and in the $C G$, only on the $6 M W T(p=0.018)$. In inter-group comparisons, it was obtained similar IMP and EMP in $\triangle I N I$, but different in $\triangle F I N(p=0.0166, p=0.0045)$ and in the $6 M W T$, there was significant difference only in $\triangle I N I$ $(p=0.0484)$. Conclusion: Isostretching and sensoriomotor aquatic training were effective at improving the FMR and the $C F$, and conventional physical therapy was effective only in the improvement of $C F$.

Keywords: Low back pain. Respiratory muscles. Exercise therapy. Proprioception.

\section{Introdução}

A dor lombar é extremamente dispendiosa, causando grande sobrecarga para o sistema de saúde. Cerca de $60 \%$ a $80 \%$ da população terá lombalgia em algum momento da vida e, destes, de $70 \%$ a $80 \%$ terão pelo menos um episódio de recorrência. Apesar da grande quantidade de recursos direcionados ao tratamento da lombalgia crônica, as intervenções terapêuticas para essa enfermidade continuam tendo baixas porcentagens de sucesso (1).

Muitos portadores de lombalgia crônica têm forte crença que suas atividades funcionais possam aumentar ainda mais a dor ou causar algum outro prejuízo físico, e esses fatores cognitivos influenciam de forma importante o nível funcional desses indivíduos. Além disso, muitos provedores de saúde aconselham que os pacientes evitem atividades que desencadeiem ou agravem a dor lombar, o que reforça as restrições de atividade. Perda das capacidades físicas, descondicionamento cardiopulmonar e muscular podem ser consequências da persistência às restrições das atividades. Essas debilidades resultam em incapacidade ou limitação para realização das atividades de vida diária (2), comprometendo a capacidade funcional, que, por definição, é a capacidade de realizar tarefas que garantam uma vida independente e o bem-estar global (3).

Uma forma segura, prática, simples e a custos baixos para se avaliar a capacidade funcional, em diversas circunstâncias clínicas, é o teste de caminhada de seis minutos (TC6), que ganhou grande importância tanto na prática clínica quanto em pesquisas nos últimos anos (4). Esse teste não é específico para nenhum sistema e avalia o indivíduo globalmente.

Embora cada sistema orgânico tenha sua contribuição na capacidade funcional, prejuízo no sistema pulmonar pode se tornar relevante entre os pacientes com lombalgia crônica. Os músculos abdominais, do diafragma e do assoalho pélvico estão relacionados 
à função ventilatória pulmonar e contribuem para a mobilidade e a estabilidade da região lombopélvica $(1,5,6)$.

Alterações no controle motor que envolvem aqueles músculos são relatadas em indivíduos com lombalgia crônica, resultando em perturbações respiratórias $(5,6)$. Além disso, especificamente, os deficits dos músculos abdominais estão associados à persistência e à cronicidade da lombalgia (7). A força desses músculos pode ser relacionada à manovacuometria, de modo que as pressões registradas na boca durante as manobras de inspiração e expiração máximas forçadas dão a noção da força muscular necessária para gerar tais pressões (8).

0 adequado controle motor dos músculos espinhais depende da informação proprioceptiva como condição prévia para coordenação e estabilidade da coluna vertebral (9). Pacientes lombálgicos crônicos apresentam mudanças na estratégia do controle motor da musculatura do tronco, que levam a prejuízos nas atividades dos músculos profundos (atraso na ativação muscular, menor tonicidade) (10) e alterações do sistema proprioceptivo, pelo alto limiar de aferências nociceptivas, contribuem para o deficit de equilíbrio postural e proprioceptivo (11).

0 isostretching é um método postural global que tem por objetivo fortalecer e flexibilizar a musculatura, corrigindo a postura e melhorando a capacidade respiratória. Os exercícios são executados com alinhamento vertebral máximo, exigindo da coluna uma atitude de autoalongamento. Assim, o método promove consciência corporal e aprimora o controle neuromuscular, a flexibilidade, a mobilidade articular, a tonicidade, a força e o controle respiratório, já que a base do método é a respiração focada principalmente na expiração (12). Embora esse recurso terapêutico seja habitualmente utilizado no meio clínico, ainda são relativamente poucos os estudos bem conduzidos que justificam o seu uso, inclusive na lombalgia crônica.

Ainda, o treino da propriocepção tem sido sugerido como um importante aspecto da intervenção no tratamento e reabilitação da lombalgia e envolve, principalmente, exercícios de equilíbrio, postura e estabilização. No entanto, um programa de reabilitação específico para melhorar a propriocepção da coluna não foi estabelecido (13). Recomenda-se que esses exercícios evoluam de condições estáticas, que não desafiam o equilíbrio, para condições de instabilidade em que as respostas de adequação de posição sejam inconscientes (14). No entanto, isso pode aumentar o risco de quedas, e o medo de cair, em si, pode ser um obstáculo ao treinamento. Dessa forma, a água pode ser uma alternativa para esse tipo de intervenção, garantindo um ambiente seguro.

A busca de formas mais eficazes para gerir a lombalgia crônica é importante quando se quer melhorar a saúde e a qualidade de vida desses pacientes (1). Muitos estudos têm demonstrado que o exercício é uma parte vital do tratamento da lombalgia crônica, mas o que ainda permanece questionável é quais são os tipos de exercícios mais benéficos (15). Assim, o objetivo deste estudo foi comparar os efeitos de duas intervenções fisioterapêuticas, sendo uma a combinação entre isostretching e treinamento sensório-motor aquático, e a outra o tratamento fisioterapêutico convencional sobre a força muscular respiratória e a capacidade funcional em portadores de lombalgia crônica.

\section{Materiais e métodos}

\section{Ética e caracterização do estudo}

Este estudo foi aprovado pelo Comitê de Ética em Pesquisa Envolvendo Seres Humanos da Universidade Estadual do Oeste do Paraná (UNIOESTE), com número de processo 019/2009, de acordo com a Resolução n. 196/96, e classifica-se como série de casos. Todos os indivíduos foram informados sobre os objetivos do estudo e assinaram o Termo de Consentimento Livre e Esclarecido antes da admissão no experimento.

\section{Caracterização da amostra e divisão dos grupos}

Foram entrevistados voluntários com diagnóstico cinesiofuncional de lombalgia crônica específica ou inespecífica, de ambos os gêneros, sedentários, encaminhados ao Centro de Reabilitação Física (CRF) da UNIOESTE, câmpus Cascavel e/ou recrutados por meio de convite formal feito nas Unidades Básicas de Saúde. Após esclarecimento dos procedimentos e objetivos da pesquisa, os voluntários foram submetidos à avaliação clínica de triagem para identificação de possíveis fatores de não inclusão, coleta da história e dos dados antropométricos, e realização do exame clínico. 0 peso e a altura foram mensurados por uma balança com estadiômetro.

Foram incluídos no estudo indivíduos: 
a) sedentários, com relato de dor lombar persistente há mais de três meses;

b) com dor lombar específica ou inespecífica cujas características clínicas e físicas fossem compatíveis com as diretrizes de avaliação e tratamento propostas pelo American College of Physicians e pelo American Pain Society (16), nas categorias 1 (portadores de lombalgia inespecífica) e 2 (portadores de lombalgia potencialmente associada à radiculopatia ou estenose espinhal);

c) com escore médio da dor dos últimos dois meses entre dois e seis pontos, previamente à intervenção, medido pela escala visual analógica (EVA).

Os critérios de não inclusão e exclusão foram:

a) indivíduos com dor lombar cujo histórico clínico sugerisse classificação na categoria 3 (dor lombar potencialmente associada à outra causa espinhal específica) das diretrizes de avaliação e tratamento propostas pelo American College of Physicians e pelo American Pain Society (16), que incluem a pequena proporção de doentes com graves ou progressivos deficits neurológicos ou condições que exijam avaliação rápida (como tumor, infecção ou síndrome da calda equina), bem como os doentes com outras condições que possam responder a tratamentos específicos, tais como a espondilite anquilosante e outras doenças reumáticas e/ ou fratura vertebral por compressão;

b) voluntários tabagistas ou ex-tabagistas por um período menor que um ano;

c) mais de $30 \%$ de falta durante o desenvolvimento da intervenção;

d) pacientes com lesões osteomusculares em outras articulações, diferenças no comprimento dos membros inferiores e doenças que comprometem a cognição;

e) realização de qualquer outro método de tratamento fisioterapêutico concomitante a este;

f) pacientes com história clínica de cirurgia na coluna, gravidez e portadores de doenças cardiovasculares que contraindiquem a prática de exercícios;

g) voluntários que não apresentassem condições hemodinâmicas compatíveis para a realização do teste de caminhada de seis minutos, hipertensos descompensados, história de cardiopatia, pneumopatias, neuropatias ou doenças dermatológicas diagnosticadas.

Inicialmente, foram entrevistados 35 voluntários com idade entre 27 e 58 anos. Destes, não foram incluídos 17 voluntários (3 por incompatibilidade de horário; 3 por lesão no sistema osteomuscular, 1 por apresentar mega apófise transversa esquerda; 1 por ter diagnóstico clínico de espondilolistese; 3 por apresentarem pneumopatias; 3 com diagnóstico clínico de doença reumática; 1 por já realizar tratamento fisioterapêutico para a dor lombar; 1 por ter realizado artrodese na coluna lombar e 1 portador de cardiopatia).

Assim, a amostra foi composta por 18 voluntários que foram distribuídos aleatoriamente, por sorteio, em dois grupos: o grupo controle (GC $\mid \mathrm{n}=08$ ), que recebeu tratamento com fisioterapia convencional, e grupo experimental (GE $\mid \mathrm{n}=10$ ), que recebeu a cinesioterapia combinada. No GC, foram excluídos três voluntários por abandono do tratamento e, no $\mathrm{GE}$, foram excluídos quatro voluntários pelo mesmo motivo e um voluntário por não apresentar condições hemodinâmicas compatíveis para a realização do TC6.

Assim, a amostra final foi composta de cinco voluntárias do gênero feminino, em cada um dos grupos, sendo que a média de idade, peso e altura para o GC foi $46,6 \pm 10,9$ anos, $72,84 \pm 20,29 \mathrm{~kg}$ e 1,62 $\pm 0,06 \mathrm{~m}$, respectivamente. Já o GE teve média de idade, peso e altura de 38,8 $\pm 7,3$ anos, $65,08 \pm 12,82 \mathrm{~kg}$ e 1,57 \pm $0,03 \mathrm{~m}$, respectivamente. Não se observou diferenças estatísticas entre essas variáveis, o que demonstra a homogeneidade da amostra.

\section{Procedimento de avaliação}

Todos os procedimentos de intervenção e avaliações foram realizados por três acadêmicos de Fisioterapia, previamente treinados, e realizados sempre nos mesmos horários do dia para evitar interferências circadianas. Antes do início da intervenção, foram realizadas as avaliações iniciais $(\Delta \mathrm{INI})$ das variáveis do estudo.

A capacidade funcional foi mensurada pelo TC6. As voluntárias foram orientadas a permanecerem sentadas por cinco minutos, visando à normalização hemodinâmica. Dados hemodinâmicos, como frequência cardíaca (FC) e pressão arterial (PA), fo- 
ram mensurados apenas para garantir que as voluntárias apresentassem condições clínicas para realizar o teste, sendo que, aquelas que apresentaram, no repouso, PA maior que 150/100 mmHg e/ou FC maior que 110 bpm foram excluídas. Como preparação para o TC6, foi realizada uma sequência de alongamentos leves com uma repetição sustentada por 30 segundos para os músculos quadríceps e isquiotibiais.

0 TC6 foi realizado em um corredor de 30 metros de comprimento, delimitados no chão por meio de uma faixa metricamente demarcada, em local plano e coberto, sobre a qual a voluntária realizava o percurso de ida e volta, quantas vezes fossem necessárias. Antes de iniciar o teste, as participantes foram instruídas quanto à execução do mesmo e alertadas para que reduzissem o ritmo de caminhada ou até mesmo interrompessem-na, caso apresentassem dores no peito, dores musculares intensas ou desconfortos respiratórios. Contudo, caso isso ocorresse, o cronômetro (marca Tenlon ${ }^{\circledR}$ ) permaneceria acionado. As voluntárias também foram orientadas a caminhar o mais rápido possível, porém sem correr, até que o examinador indicasse o momento de parada, quando transcorridos os seis minutos. Durante toda a caminhada, em intervalos de 30 segundos, o examinador proferiu frases de incentivo tais como "Continue assim", "Vamos lá, você está indo bem". Durante a realização do teste, o examinador caminhou, discretamente, atrás e não ao lado de cada participante, para não influenciar o ritmo da caminhada. Ao fim dos seis minutos, foi feito o registro da distância percorrida por cada voluntária.

A partir dos dados antropométricos coletados na avaliação de triagem, foram calculadas as distâncias preditivas para idade, gênero feminino, altura e peso de cada voluntária, consideradas como valores de referência (TC6 REF), por meio da seguinte fórmula proposta na literatura (17): distância prevista $(\mathrm{m})=$ $(2,11 \times$ altura $\mathrm{cm})-(5,78 \times$ idade $)-(2,29 \times$ peso $\mathrm{kg})$ $+667 \mathrm{~m}$.

A força muscular respiratória foi avaliada, indiretamente, com um manovacuômetro da marca Ger-Ar ${ }^{\circledR}$ pela mensuração das pressões inspiratória e expiratória máximas (Pimáx e Pemáx, respectivamente), considerando-se que quanto maiores as pressões geradas, maior a força muscular respiratória. A participante foi orientada a realizar uma inspiração máxima a partir do volume residual, para medir a Pimáx. Já para a determinação da Pemáx, realizou-se uma expiração máxima a partir da capacidade pulmonar total. Nos dois testes, foram registradas as pressões de pico (18). Para ambos os testes, realizaram-se de três a cinco tentativas e o maior valor foi considerado, registrado em $\mathrm{cmH}_{2} \mathrm{O}$. Caso o maior valor aparecesse na última tentativa, o teste era repetido até que fosse reproduzido um valor inferior. Como a realização do teste era cansativa, foi concedido à voluntária um intervalo de um minuto entre as manobras.

Os valores previstos para as pressões respiratórias máximas foram calculados por meio das equações de predição propostas por Neder et al. (19), em função do gênero e idade das participantes. Essas equações foram descritas por Souza (20), nas Diretrizes para Testes de Função Pulmonar, publicadas oficialmente pela Sociedade Brasileira de Pneumologia e Tisiologia; e, para cada uma das variáveis, o limite inferior de normalidade foi calculado subtraindo-se o valor previsto pelo produto calculado de: 1,65 $\times$ EPE (erro padrão de estimativa). Assim, foram utilizadas as seguintes equações (equação de predição associada ao cálculo do limite inferior de normalidade): PImáx $=(110,4-[0,49 \times$ idade $])-(1,65 \times$ EPE $)$, com $\mathrm{EPE}=9,1$ e PEmáx $=(115,6-[0,61 \times$ idade $])-(1,65$ $\times \mathrm{EPE})$, com EPE = 11,2.

Para todas as variáveis, a avaliação final ( $\Delta$ FIN) foi realizada após o término da intervenção.

\section{Procedimento de intervenção}

As intervenções foram realizadas com frequência de três sessões semanais, durante sete semanas consecutivas, com duração média de uma hora cada sessão. Previamente ao início da intervenção, as voluntárias foram reunidas e todos os detalhes dos exercícios foram explicados.

As voluntárias pertencentes ao GC receberam tratamento conservador composto por eletroterapia analgésica (com Estimulação Nervosa Elétrica Transcutânea - TENS da marca Bioset ${ }^{\circledR}$, com frequência de $80 \mathrm{~Hz}$ e duração do pulso de $250 \mu$ s, sendo que os eletrodos foram posicionados nos músculos paravertebrais lombares e aplicados durante $15 \mathrm{mi}$ nutos), massoterapia na região lombar (composta pelas técnicas de deslizamento superficial e profundo realizadas durante um minuto, evoluindo com técnicas de pétrissage realizadas por quatro minutos) e alongamentos segmentares bilaterais para os músculos isquiotibiais, paravertebrais e músculos laterais do tronco, os quais foram sustentados por 30 segundos, repetidos por duas vezes. 
As voluntárias pertencentes ao GE foram submetidas à intervenção com técnica isostretching em solo nos 30 minutos iniciais e, em seguida, realizaram o treinamento sensório-motor aquático por mais 30 minutos. Previamente à aplicação da intervenção, foi realizada uma sessão destinada a ensinar quesitos básicos para a execução correta das posturas: estabilização segmentar, que potencializa o padrão neuromuscular e aumenta a estabilidade espinhal, melhorando a resistência dos músculos abdominais profundos; o alinhamento adequado da coluna para as posturas, entendido como retificação das curvaturas vertebrais; a respiração prolongada e o autoalongamento $(12,21,22)$.

A seleção das posturas foi adaptada dentre aquelas descritas na literatura e seguiu as seguintes orientações:

a) em cada sessão foram escolhidas de seis a sete posturas dentre todas as posturas descritas pelo criador do método isostretching (23);

b) fez-se um rodízio das posturas, todas simétricas para tornar mais fácil a manutenção da postura, que foram executadas nas posições ortostática (duas posturas), em decúbito dorsal (de duas a três posturas) e sentada (de duas a três posturas);

c) as posturas selecionadas enfocaram prioritariamente as cadeias respiratória, ântero-interna do quadril e cadeia mestra posterior;

d) a partir da décima sessão, graus de dificuldades foram implementados quando possível, respeitando-se a individualidade das participantes, pela utilização de bastões e de bolas leves;

e) o tempo de manutenção de cada exercício foi determinado pelo tempo de expiração desenvolvida por meio da projeção semicerrada dos lábios, a qual durou cerca de dez segundos;

f) cada postura foi realizada três vezes, com intervalo de 15 segundos entre as repetições, sendo que a primeira repetição destinou-se à compreensão, a segunda à correção e a terceira à execução da melhor maneira possível;

g) em todas as posturas realizadas, independente do grau de dificuldade, a prioridade foi dada para o alinhamento correto da coluna vertebral, mesmo que isso significasse que os membros não seriam estirados e mantidos na amplitude de movimento máxima durante a execução; h) cada postura foi previamente demonstrada pelo pesquisador/instrutor antes da execução pelas voluntárias;

i) o pesquisador/instrutor corrigiu, por meio de estímulo verbal e/ou tátil, as voluntárias que eventualmente não estivessem executando o exercício da forma correta (12).

No treinamento sensório-motor aquático, foi realizada uma sessão piloto da intervenção, para facilitar o aprendizado, que não foi contabilizada como efetiva. A intervenção teve seus níveis de complexidade modificados de acordo com a evolução proprioceptiva das voluntárias, mas foi pautada em três condições básicas: fase estática, na qual foram priorizados os exercícios de estabilização pélvica com alinhamento dos pés e coluna cervical; fase dinâmica, na qual as voluntárias desenvolveram a habilidade de manter a estabilização pélvica em uma gama de situações que desafiaram seu centro de gravidade; fase funcional, em que foram inseridas atividades que desafiaram o controle postural, compostas por condições que utilizaram apoios monopodálicos, bipodálicos, desequilíbrios provocados pelo terapeuta ou pelo exercício, variações de intensidade e superfícies, privação da visão, deslocamentos em todas as direções, aceleração e desaceleração, e exercícios em cadeias cinéticas (fechada e aberta), além de exercícios de coordenação muscular (14).

A intervenção aquática foi realizada na piscina terapêutica aquecida da Clínica de Fisioterapia da UNIOESTE, cujas dimensões são de 7,80 metros de largura, 11,80 metros de comprimento, 1,00 metro de profundidade nos primeiros 5,90 metros de comprimento, onde foi realizada a intervenção, e 60 centímetros de profundidade nos outros 5,90 metros. Foi realizado um aquecimento prévio antes de cada sessão, com trotes leves.

\section{Tratamento estatístico}

Os dados referentes à Pimáx e Pemáx foram normalizados dividindo-se o valor de teste pelo valor predito pelo limite inferior. O TC6 também foi normalizado pelo valor predito. A normalização facilita a visualização do quanto o valor de teste se aproximou daquele predito, de forma que: razão $<1$ indicou que o indivíduo não atingiu o valor predito; razão $=1$ indicou que o valor de teste foi igual ao 
predito; e razão $>1$, que o valor de teste superou 0 valor predito.

Para o tratamento estatístico, utilizou-se o software GraphPad Prism 3.0. Em função do baixo "n" amostral, as comparações foram feitas por testes não paramétricos. Nas comparações intergrupo, utilizou-se o teste de Mann-Whitney e para as comparações intragrupo, o teste de Wilcoxon.

Os dados antropométricos, a idade e os dados da $(\triangle \mathrm{INI})$ para o TC6, Pimáx e para a Pemáx dos dois grupos foram comparados pelo teste de MannWhitney. 0 intuito dessas comparações foi verificar a homogeneidade da amostra.

Para todos os testes comparativos descritos acima, foi adotado $(\alpha=0,05)$.

\section{Resultados}

Neste estudo, observou-se melhora significativa para todas as variáveis no GE nas comparações intragrupo. Já no GC, houve diferença significativa somente na capacidade funcional. Os valores médios normalizados de cada variável em ambos os grupos, os desvios-padrão e a estatística inferencial podem ser visualizados no Gráfico 1.

No Gráfico 2, podem ser visualizadas as comparações intergrupo. As variáveis Pimáx e Pemáx, tanto no GC como no GE, nas $\triangle I N I$ foram estatisticamente semelhantes e nas $\Delta$ FIN foram significativamente diferentes. Verifica-se que os grupos partiram da mesma condição pulmonar antes da intervenção, mas se tornaram diferentes ao fim do experimento. Quanto ao TC6, nas $\triangle \mathrm{INI}$ houve diferença significativa entre os grupos, sendo que o GC partiu de melhor capacidade funcional. Embora ambos os grupos tenham melhorado a capacidade funcional (comparações intragrupo), a diferença entre os grupos não foi significativa nas $\Delta$ FIN.

\section{Discussão}

A análise dos resultados obtidos neste trabalho sugere que a capacidade funcional pode ser aprimorada pela aplicação da fisioterapia convencional e da cinesioterapia combinada.

O TC6 é indicador da capacidade física geral e da mobilidade. Assim, fatores como força, equilíbrio, velocidade, entre outros, podem influenciar o desempenho

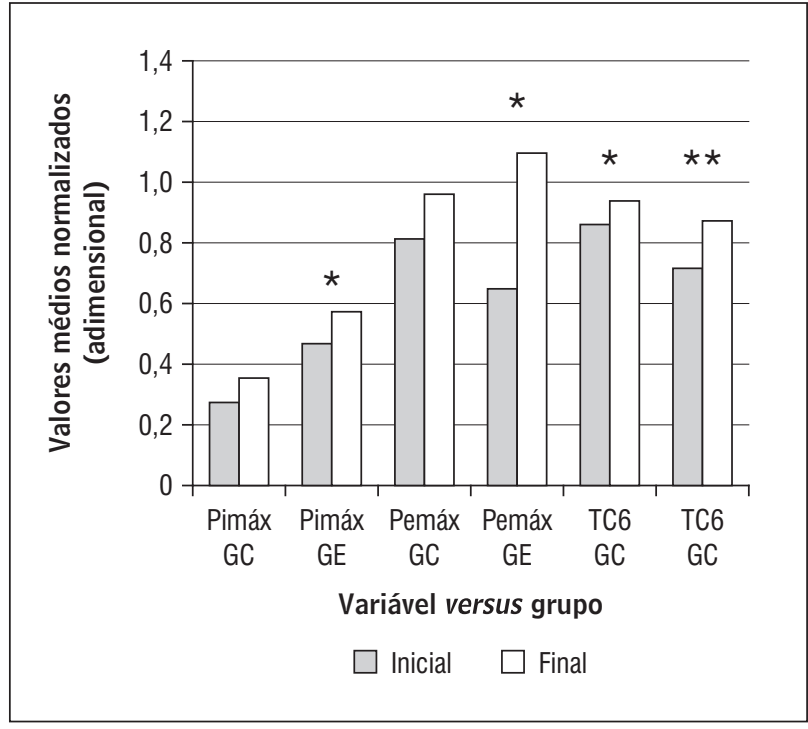

Gráfico 1 - Comparações intragrupo entre as variáveis Pimax, Pemax e TC6

Legenda: Pressão inspiratória máxima = Pimáx; pressão expiratória máxima $=$ Pemáx; teste de caminhada de seis minutos = TC6; grupo controle = GC; grupo experimental = GE. Os asteriscos representam os níveis de significância encontrados $\left({ }^{*} p<0,01 ;{ }^{*} p<0,001\right)$

Fonte: Dados da pesquisa.

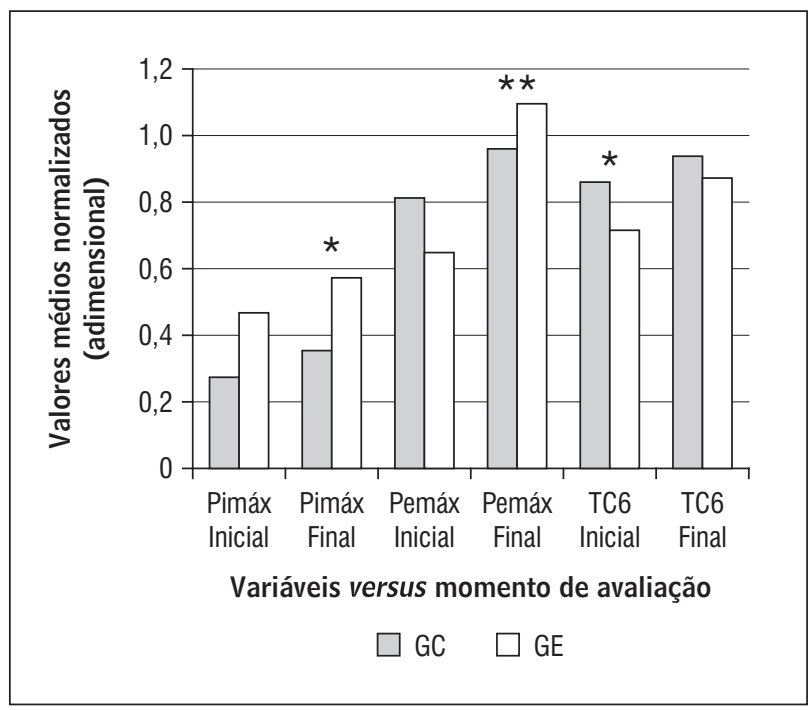

Gráfico 2 - Comparações intergrupo entre as variáveis Pimax, Pemax e TC6

Legenda: Pressão inspiratória máxima = Pimáx; pressão expiratória máxima = Pemáx; avaliação inicial = I; avaliação final $=\mathrm{F}$; teste de caminhada de seis minutos = TC6; grupo controle $=\mathrm{GC}$; grupo experimental $=\mathrm{GE}$. Os asteriscos representam os níveis de significância encontrados $\left({ }^{*} p<0,01 ;{ }^{*} p<0,001\right)$.

Fonte: Dados da pesquisa. 
no teste (24). Como o TC6 não é capaz de identificar quais habilidades e/ou fatores contribuíram para o melhor desempenho de ambos os grupos no presente trabalho, acredita-se que, no GE, em função do tipo de intervenção aplicada, os sistemas musculoesquelético, cardiopulmonar e nervoso estiveram envolvidos no aprimoramento da capacidade funcional (12).

0 isostretching promove adaptações funcionais por meio das contrações isométricas e excêntricas, como a melhora da força e da resistência muscular (25), promove maior ativação e controle neuromuscular abdominal, melhorando a estabilização do tronco e, consequentemente, o desempenho na marcha (12).

A atividade física na água aumenta o equilíbrio, a coordenação e estimula o sistema vestibular. Os estímulos térmicos e hidromecânicos associados aos efeitos dos princípios físicos da água promovem relaxamento muscular, reduzem a intensidade da dor (26), permitem maior mobilidade articular pela redução do estresse sobre as articulações (27) e melhoram o mecanismo de controle postural (26). Assim, os benefícios decorrentes da aplicação da cinesioterapia combinada podem justificar o aprimoramento da capacidade funcional neste grupo.

A eletroanalgesia, a massagem e o alongamento são técnicas convencionais utilizadas para o tratamento da dor lombar crônica. No estudo de Poitras e Brosseau (28), os portadores de lombalgia crônica melhoraram as limitações físicas, emocionais e a dor após o uso da TENS. A massagem, por sua vez, melhora os sintomas e a função, já que a manipulação da fáscia e dos músculos induzem alterações bioquímicas locais, modulam o fluxo sanguíneo, a oxigenação local e aumentam o limiar da dor por meio da liberação de endorfinas e serotonina (29). 0 alongamento reduz a deficiência e a dor de portadores de lombalgia (30). Sugere-se então que, no presente estudo, a associação das modalidades aplicadas no GC favoreceu a redução da dor, repercutindo na melhora da capacidade funcional desse grupo.

Outro fator que pode ter influenciado o desempenho no TC6, em ambos os grupos, é o psicológico (31). A literatura sugere que as melhoras da capacidade funcional indicam tanto o aprimoramento da aptidão física quanto melhora no domínio psicológico (32).

Há evidências na literatura de que o treinamento da musculatura respiratória melhora a capacidade funcional, especificamente na doença pulmonar obstrutiva crônica (DPOC). 0 estudo de Hill et al. demonstrou que, após oito semanas de treinamento muscular inspiratório, indivíduos com DPOC obtiveram melhoras significativas na força muscular inspiratória, na resistência muscular inspiratória, dispneia e fadiga, e ganhos modestos na capacidade funcional verificada pelo TC6 (33). De forma similar, outro estudo que utilizou espirometria de incentivo a fluxo para treinar a musculatura respiratória, também encontrou aumento significativo da distância percorrida em seis meses em relação ao controle (34). Da mesma forma que os portadores de DPOC melhoraram a capacidade funcional pelo treinamento muscular respiratório, os dados do presente estudo sugerem a interdependência dessas variáveis também em portadores de lombalgia crônica.

Moreno et al. (35) analisaram o efeito do método de Reeducação Postural Global (RPG), técnica postural similar ao isostretching, sobre a força muscular respiratória de homens jovens sedentários. Os valores da Pimáx e Pemáx do grupo controle não apresentaram diferença, mas o grupo RPG obteve valores significativamente maiores após o treinamento.

0 método isostretching enfatiza o trabalho respiratório por meio da expiração forçada e prolongada desenvolvida pela projeção semicerrada dos lábios, promovendo recrutamento ativo da musculatura abdominal, sendo efetivo sobre a função pulmonar em virtude da melhora da atuação diafragmática e da biomecânica respiratória (36). Além disso, o treinamento sensório-motor aquático pode ter favorecido a melhora da força muscular respiratória em virtude dos efeitos da pressão hidrostática, os quais dificultam a inspiração e favorecem a expiração, exigindo maior trabalho dos músculos respiratórios (37). Estes também sofrem adaptação ao treinamento muscular, como a melhora da sincronia no recrutamento das unidades motoras, gerando melhor coordenação da contração muscular e, consequentemente, maior tensão tecidual (38).

O GC não obteve melhora nas pressões respiratórias máximas. Isso pode ser justificado pelo fato de que o protocolo aplicado nesse grupo não teve abordagens relacionadas diretamente ou indiretamente ao sistema pulmonar.

Como limitação do estudo, encontrou-se a dificuldade de se descrever e seguir um protocolo específico, já que o isostretching possui várias posturas, todas ricas em detalhes de posicionamento, o que tornaria complexa a sua descrição. Em razão do baixo "n" amostral, não foi possível a realização de grupos 
isolados para se conhecer os reais efeitos e a contribuição de cada técnica nas respostas observadas neste estudo. Assim, pesquisas futuras devem ser realizadas para se conhecer os mecanismos de ação que apoiem seu uso e que elucidem quais tecidos e como eles são influenciados por cada técnica isolada.

\section{Conclusões}

Os resultados reportados neste estudo evidenciam que a combinação entre isostretching e treinamento sensório-motor aquático foi eficaz na melhora da capacidade funcional e na força dos músculos respiratórios em portadores de lombalgia crônica, enquanto o tratamento fisioterapêutico clássico foi efetivo apenas na melhora da capacidade funcional.

\section{Referências}

1. Macedo LG, Latimer J, Maher CG, Hodges PW, Nicholas $\mathrm{M}$, Tonkin L, et al. Motor control or graded activity exercises for chronic low back pain? A randomised controlled trial. BMC Musculoskelet Disord. 2008;9:65. doi:10.1186/1471-2474-9-65

2. Cohen I, Rainville J. Aggressive exercise as treatment for chronic low back pain. Sports Med. 2002;32(1):75-82.

3. Malmberg JJ, Miilunpalo SI, Vuori IM, Pasanen ME, Oja P, Haapanen-Niemi NA. A health-related fitness and functional performance test battery for middle-age and older adults: feasibility and health-related content validity. Arch Phys Med Rehabil. 2002;83(5):666-77.

4. ATS Committee on Proficiency Standards for Clinical Pulmonary Function Laboratories. ATS Statement: guideline for the six-minute walk test. Am J Respir Crit Care Med. 2002;166(1):111-7. doi: 10.1164/ rccm.166/1/111

5. Roussel NA, Nijs J, Truijen S, Smeuninx L, Stassijns G. Low back pain: clinimetric properties of the Trendelenburg test, active straight leg raise test, and breathing pattern during active straight leg raising. J Manipulative Physiol Ther. 2007;30(4):270-8.

6. O'Sullivan PB, Beales DJ, Beetham JA, Cripps J, Graf F, Lin I, et al. Altered motor control strategies in subjects with sacroiliac joint pain during the active straightleg-raise test. Spine. 2002;27(1):E1-8.
7. Hall L, Tsao H, MacDonald D, Coppieters M, Hodges PW. Immediate effects of co-contraction training on motor control of the trunk muscles in people with recurrent low back pain. J Electromyogr Kinesiol. 2009;19(5):763-73. doi:10.1016/j.jelekin.2007.09. 008

8. ATS/ERS Statement on Respiratory Muscle Testing. Am J Respir Crit Care Med. 2002;166:518-624.

9. Hjortskov N, Hye-Knudsen C, Fallentin N. Lumbar position sense acuity during an electrical shock stressor. BMC Musculoskelet Disord. 2005;6:37. doi: 10.1186/1471-2474-6-37

10. Maher CG, Latimer J, Hodges PW, Refshauge KM, Moseley LG, Herbert RD, et al. The effect of motor control exercise versus placebo in patients with chronic low back pain. BMC Musculoskelet Disord. 2005;6:54 doi:10.1186/1471-2474-6-54

11. Sung PS, Park HS. Gender differences in ground reaction force following perturbations in subjects with low back pain. Gait Posture. 2009;29(2):290-5.

12. Carvalho AR, Assini TCKA. Aprimoramento da capacidade funcional de idosos submetidos a uma intervenção por isostretching. Rev Bras Fisioter. 2008; 12(4):269-73.

13. Petersen CM, Zimmermann CL, Cope S, Bulow ME, Panveno EE. A new measurement method for spine reposition sense. J Neuroeng Rehabil. 2008;5:9. doi: 10.1186/1743-0003-5-9

14. Page P. Sensoriomotor training: a "global" approach for balance training. J Bodyw Mov Ther. 2006;10:77-84.

15. Barr KP, Griggs M, Cadby T. Lumbar stabilization: a review of core concepts and current literature, part 2. Am J Phys Med Rehabil. 2007;86(1):72-80.

16. Chou R, Qaseem A, Snow V, Casey D, Cross JT Jr, Shekelle $\mathrm{P}$, et al. Diagnosis and treatment of low back pain: a joint clinical practice guideline from the American College of Physicians and the American Pain Society. Ann Intern Med. 2007;147(7):478-91.

17. Enright PL, Sherril DL. Reference equations for the six-minute walk in healthy adults. Am J Respir Crit Care Med. 1998;158(5 Pt 1):1384-7.

18. Black LF, Hyatt RE. Maximal respiratory pressures: normal values and relationship to age and sex. Am Rev Respir Dis. 1969;99(5):696-702. 
19. Neder JA, Andreoni S, Lerario MC, Nery LE. Reference values for lung function tests II. Maximal respiratory pressure and voluntary ventilation. Braz J Med Biol Res. 1999;32(6):719-27.

20. Souza RB. Pressões respiratórias estáticas máximas. Diretrizes para testes de função pulmonar. J Pneumol. 2002;28(Suppl 3):S155-65.

21. Hicks GE, Fritz JM, Delitto A, McGill M. Preliminary development of a clinical prediction rule for determining which patients with low back pain will respond to a stabilization exercise program. Arch Phys Med Rehabil. 2005;86(9):1753-62.

22. Cairns MC, Foster NE, Wright C. Randomized controlled trial of specific spinal stabilization exercises and conventional physiotherapy for recurrent low back pain. Spine. 2006;31(19):E670-81.

23. Redondo B. Iso-Stretching. Foz do Iguaçu: [s.n.]; 2008. [Apostila do Curso de Iso-Stretching].

24. Lord SR, Menz HB. Physiologic, psychologic, and health predictors of 6-minute walk performance in older people. Arch Phys Med Rehabil. 2002;83(7):907-11.

25. Blazevich AJ. Effects of physical training and detraining, immobilization, growth and aging on human fascicle geometry. Sports Med. 2006;36(12):1003-17.

26. Melzer I, Elbar 0, Tsedek I, Oddsson LIE. A water-based training program that include perturbation exercises to improve stepping responses in older adults: study protocol for a randomized controlled cross-over trial. BMC Geriatr. 2008;8:19. doi:10.1186/1471-2318-8-19

27. Devereux K, Robertson D, Briffa NK. Effects of a water-based program on women 65 years and over: a randomised controlled trial. Aust J Physiother. 2005;51(2):102-8.

28. Poitras S, Brosseau L. Evidence-informed management of chronic low back pain with transcutaneous electrical nerve stimulation, interferential current, electrical muscle stimulation, ultrasound, and thermotherapy. Spine J. 2008;8(1):226-33.

29. Imamura M, Furlan AD, Dryden T, Irvin E. Evidenceinformed management of chronic low back pain with massage. Spine J. 2008;8(1):121-33.
30. Cleland JA, Childs JD, Palmer JA, Eberhart S. Slump stretching in the management of non-radicular low back pain: a pilot clinical trial. Man Ther. 2006;11(4): 279-86.

31. Reneman MF, Jan H, Geertzen B, Groothoff JW, Brouwer S. General and specific self-efficacy reports of patients with chronic low back pain: are they related to performances in a functional capacity evaluation? J Occup Rehabil. 2008;18(2):183-9.

32. Koumantakis GA, Watson PJ, Oldham JA. Supplementation of general endurance exercise with stabilisation training versus general exercise only physiological and functional outcomes of a randomised controlled trial of patients with recurrent low back pain. Clin Biomech. 2005;20(5):474-82.

33. Hill K, Jenkins SC, Philippe DL, Cecins N, Shepherd KL, Green DJ, et al. High-intensity inspiratory muscle training in COPD. Eur Respir J. 2006;27(6):1119-28.

34. Crisafulli E, Costi S, Fabbri LM, Clini EM. Respiratory muscles training in COPD patients. Int J Chron Obstruct Pulmon Dis. 2007;2(1):19-25.

35. Moreno MA, Catai AM, Teodori RM, Borges BLA, Cesar $\mathrm{MC}$, Silva E. Efeito de um programa de alongamento muscular pelo método de Reeducação Postural Global sobre a força muscular respiratória e a mobilidade toracoabdominal de homens jovens sedentários. J Bras de Pneumol. 2006;33(6):679-86.

36. Brandt AC, Ricieri DV, Griesbach LE. Repercussões respiratórias da aplicação da técnica de isostretching em indivíduos sadios. Fisioter Bras. 2004;5(2):103-10.

37. Caromano FA, Themudo MRF Filho, Candeloro JM. Efeitos fisiológicos da imersão e do exercício na água. Fisioter Bras. 2003;4(1):1-5.

38. Powers SK, Howley ET. Fisiologia do exercício. 3a ed. São Paulo: Manole; 2000.

Recebido: 01/03/2011 Received: 03/01/2011

Aprovado: 01/08/2011 Approved: 08/01/2011 